\title{
Research Article Efficient Recursive Multichannel Blind Image Restoration
}

\author{
Li Chen, Kim-Hui Yap, and Yu He \\ Division of Information Engineering, School of Electrical and Electronic Engineering, Nanyang Technological University, \\ 50 Nanyang Avenue, Singapore 639798
}

Received 3 May 2006; Revised 25 August 2006; Accepted 26 August 2006

Recommended by Mark Liao

This paper presents a novel multichannel recursive filtering (MRF) technique to address blind image restoration. The primary motivation for developing the MRF algorithm to solve multichannel restoration is due to its fast convergence in joint blur identification and image restoration. The estimated image is recursively updated from its previous estimates using a regularization framework. The multichannel blurs are identified iteratively using conjugate gradient optimization. The proposed algorithm incorporates a forgetting factor to discard the old unreliable estimates, hence achieving better convergence performance. A key feature of the method is its computational simplicity and efficiency. This allows the method to be adopted readily in real-life applications. Experimental results show that it is effective in performing blind multichannel blind restoration.

Copyright ( 2007 Hindawi Publishing Corporation. All rights reserved.

\section{INTRODUCTION}

Image restoration deals with the estimation of the original images from the observed blurred, degraded images using the partial information about the imaging system. It is an illposed problem as the uniqueness and stability of the solution are not guaranteed [1]. In many applications such as remote sensing and microscopy imaging, multiple degraded images of a single scene become available while the blurring function or point spread function (PSF) of each channel remains unknown. Therefore, the recovery of the original scene from its multiple observations is required and this problem is, commonly, referred to as multichannel blind image restoration [2].

Various researchers have investigated the problem of multichannel image restoration over the years. With the assumption that the multichannel PSFs are weakly coprime, and in the absence of noise, the desired image and PSFs can be transformed into the null space of a special matrix constructed from the degraded images [3-6]. Centered on this idea, several techniques have been proposed which include greatest common divisor (GCD) [3], subspace-based [4, 5], and eigenstructure-based approaches [6]. The GCD method is based on the notion that the desired image can be regarded as the polynomial GCD among the degraded images in the $z$-domain. Subspace-based methods work by first estimating the blurring function using a procedure of min-eigenvector, followed by conventional image restoration using the identi- fied PSFs. In similar concept, eigenstructure-based algorithm transforms the null space problem into a constrained optimization framework and performs direct deconvolver estimation. The aforementioned null space-based methods, however, suffer from noise amplification, which often lead to poor solutions in the noisy environments.

There are some successful works on the development of multichannel restoration, which exploit the features of single-channel restoration algorithms [7-15]. These techniques develop a cost function within the framework of constrained least squares minimization $[7,8]$. The minimization step involves two processes of blur identification and image restoration centered on the principle of projection onto convex sets (POCS). The alternating minimization (AM) strategy is first proposed in [9], and later extended to double Tikhonov regularization in $[10,11]$. Double regularization (DR) [12] and the Gauss-Markov random fields [13] have also been applied in blind image restoration. Total variation (TV) has been incorporated into the DR to achieve edge preservation and noise suppression. A promising attempt has been made by utilizing the blur null space as the regularization term in the framework of TV [14]. Recently, the extension of the Bussgang blind equalization algorithm to iterative multichannel deconvolution has been proposed in [15]. The basic idea is focused on Wiener filtering of the observed degraded images, and updating the filters using a nonlinear Bayesian estimation of the estimated image. Generally speaking, these iterative methods are extensions of 
single-channel blind image restorations approaches. Therefore, if an extra degraded image becomes available at a later stage, the iterative schemes will require a complete rerun, rather than a recursive process to update the estimate. This is, clearly, inflexible and computationally inefficient.

In view of this, we develop a new efficient algorithm called multichannel recursive filtering (MRF) to solve blind multichannel image restoration. To the best of our knowledge, no previous work on recursive filtering has been developed to address blind multichannel image restoration. The estimated image is recursively updated from the previous estimate using a regularization framework. All the operations of MRF are performed in discrete Fourier transform (DFT) domain, giving rise to fast and simple implementation. The multichannel PSFs are identified iteratively using the conjugate gradient optimization. The proposed algorithm incorporates a forgetting factor to discard the old unreliable estimates, hence achieving better convergence performance. A key feature of the method is its computational simplicity and efficiency. This allows the new method to be adopted readily in real-life applications.

The rest of this paper is organized as follows. Section 2 provides a brief discussion on the multichannel blind restoration problem. In Section 3, the development of recursive image restoration algorithm is presented. In Section 4, issues related to the selection of regularization parameters and forgetting factor are discussed. Simulation results are given in Section 5. In Section 6, conclusions and further remarks are drawn.

\section{PROBLEM FORMULATION}

In this work, we are interested in the single-input multipleoutput (SIMO) multichannel blind image restoration problem. Considering the SIMO linear degradation system that consists of $K$ measurements of the original image $\mathbf{f}$, the observed degraded image of the $i$ th-channel can be modeled as [3-6]:

$$
\mathbf{g}_{i}=\mathbf{h}_{i} * \mathbf{f}+\mathbf{n}_{i}, \quad i=1,2, \ldots, K
$$

where $*$ denotes two-dimensional (2D) convolution, $\mathbf{g}_{i}, \mathbf{h}_{i}$, and $\mathbf{n}_{i}$ represent the degraded image, PSF, and noise of the $i$ th-channel, respectively. To tackle the ill-posed nature of image restoration, Tikhonov-Miller regularization theory has been employed in the restoration scheme, as it is effective in edge preservation and noise suppression. The regularization principle has been extended to a double regularization framework that imposes smoothness constraint in both the image- and blur domains. In terms of the $i$ th-channel, it can be described by [7-12]:

$$
\begin{gathered}
\left\|\mathbf{g}_{i}-\mathbf{h}_{i} * \mathbf{f}\right\|^{2}=\left\|\mathbf{n}_{i}\right\|^{2} \leq \varepsilon_{i}^{2} \\
\|\mathbf{c} * \mathbf{f}\|^{2} \leq \gamma^{2}, \quad\left\|\mathbf{d}_{i} * \mathbf{h}_{i}\right\|^{2} \leq \delta_{i}^{2}
\end{gathered}
$$

where $\|\cdot\|$ represents the $L_{2}$-norm. $\varepsilon_{i}, \gamma, \delta_{i}$ are the upper bounds related to the noise, image, and PSF terms, respectively. $\mathbf{c}$ and $\mathbf{d}_{i}$ are the regularization operators and usually take the form of a high-pass filter. The first term in (2) is the data-fidelity term, while the second and third terms are the regularization functionals that impose smoothness constraints on the image- and blur-domains, respectively. In order to perform joint blur identification and image restoration for multichannel problem, the following cost function is formulated over $K$ channels:

$$
J(\mathbf{f}, \mathbf{h})=\sum_{i=1}^{K}\left(\left\|\mathbf{g}_{i}-\mathbf{h}_{i} * \mathbf{f}\right\|^{2}+\alpha_{i}\|\mathbf{c} * \mathbf{f}\|^{2}+\beta_{i}\left\|\mathbf{d}_{i} * \mathbf{h}_{i}\right\|^{2}\right),
$$

where $\mathbf{h}=\left\{\mathbf{h}_{1}, \ldots, \mathbf{h}_{K}\right\}$ is the multichannel blurs. The cost function in (3) consists of the data-fidelity term, and the image- and blur-domain regularization terms. $\alpha_{i}$ and $\beta_{i}$ are the regularization parameters that offer a compromise between least-square fidelity error and the regularity of the solutions $\mathbf{f}$ and $\mathbf{h}_{i}$. As it is computationally intensive to perform joint optimization to estimate $\mathbf{f}$ and $\mathbf{h}$ simultaneously, an iterative strategy based on alternating minimization (AM) is adopted to project the overall cost function $J(\mathbf{f}, \mathbf{h})$ into the image-domain cost function $J(\mathbf{f} \mid \mathbf{h})$ and the blur-domain cost function $J(\mathbf{h} \mid \mathbf{f})$. It can be shown that $J(\mathbf{f} \mid \mathbf{h})$ and $J(\mathbf{h} \mid \mathbf{f})$ are quadratic with positive semidefinite Hessian matrices. This suggests that the cost function in each domain is convex, hence ensuring the convergence in their respective domains. In conventional iterative multichannel blind restoration algorithms [12-14], if a new degraded image (i.e., $(K+1)$-th measurement $\left.\mathbf{g}_{K+1}\right)$ becomes available at a later stage, the iterative schemes will require a complete rerun, hence incurring a significant computational cost. The proposed method strives to alleviate this difficulty by developing a recursive algorithm to update the estimate, thereby reducing the computational cost.

\section{RECURSIVE FILTERING FOR MULTICHANNEL BLIND IMAGE RESTORATION}

\subsection{Multichannel recursive filtering (MRF)}

The overall cost function in (3) consists of two sets of unknown variables: image and blur. As explained earlier, we project $J(\mathbf{f}, \mathbf{h})$ into the image-domain cost function $J(\mathbf{f} \mid \mathbf{h})$ by fixing the blurring filters $\mathbf{h}$ to give

$$
J(\mathbf{f} \mid \mathbf{h})=\sum_{i=1}^{K}\left(\left\|\mathbf{g}_{i}-\mathbf{h}_{i} * \mathbf{f}\right\|^{2}+\alpha_{i}\|\mathbf{c} * \mathbf{f}\|^{2}\right) .
$$

It is observed that (4) is a convex function with respect to f. Recursive filtering is widely used in 1D signal processing due to its fast convergence rate, as compared with least mean squares (LMS) filtering [16]. Motivated by this consideration, we develop a new multichannel recursive filtering (MRF) scheme to address the 2D problem in this work. It is worth noting that, unlike the $1 \mathrm{D}$ cases where matrix inversion lemma is used in the development of recursive least square filtering, the same approach cannot be applied directly in $2 \mathrm{D}$ images due to significantly increased complexity. In view of this, we propose an MRF scheme that utilizes 2D-DFT to update the image estimate. This formulation of MRF is outlined as follows. 
(i) Initialize the algorithm by setting

$$
\mathbf{f}^{(0)}=\mathbf{0}, \quad \widetilde{\mathbf{R}}^{(0)}=\mathbf{0}, \quad \lambda \leq 1 .
$$

(ii) For the $(n+1)$ th channel $n=0,1, \ldots, K-1$, set $\alpha_{n+1}, \mathbf{c}_{n+1}$, and calculate the 2D-DFT ${ }^{a}$ :

$$
\tilde{\mathbf{f}}^{(n)}=\mathcal{F}\left[\mathbf{f}^{(n)}\right], \quad \tilde{\mathbf{g}}_{n+1}=\mathcal{F}\left[\mathbf{g}_{n+1}\right], \quad \tilde{\mathbf{H}}_{n+1}=\mathcal{F}\left[\mathbf{h}_{n+1}\right], \quad \widetilde{\mathbf{C}}_{n+1}=\mathcal{F}\left[\mathbf{c}_{n+1}\right] .
$$

Update the old estimate recursively:

$$
\tilde{\mathbf{f}}^{(n+1)}(\boldsymbol{\omega})=\tilde{\mathbf{f}}^{(n)}(\boldsymbol{\omega})+\frac{\tilde{\mathbf{H}}_{n+1}^{H}(\boldsymbol{\omega}) \tilde{\mathbf{g}}_{n+1}(\boldsymbol{\omega})-\left(\left|\widetilde{\mathbf{H}}_{n+1}(\boldsymbol{\omega})\right|^{2}+\alpha_{n+1}\left|\widetilde{\mathbf{C}}_{n+1}(\boldsymbol{\omega})\right|^{2}\right) \tilde{\mathbf{f}}^{(n)}(\boldsymbol{\omega})}{\widetilde{\mathbf{R}}^{(n+1)}(\boldsymbol{\omega})},
$$

where $\widetilde{\mathbf{R}}^{(n+1)}(\boldsymbol{\omega})=\lambda \widetilde{\mathbf{R}}^{(n)}(\boldsymbol{\omega})+\left|\widetilde{\mathbf{H}}_{n+1}(\boldsymbol{\omega})\right|^{2}+\alpha_{n+1}\left|\widetilde{\mathbf{C}}_{n+1}(\boldsymbol{\omega})\right|^{2}$.

Calculate the inverse DFT:

$$
\mathbf{f}^{(n+1)}=\mathcal{F}^{-1}\left[\widetilde{\mathbf{f}}^{(n+1)}\right]
$$

${ }^{a}$ In Matlab, the 2D-DFT of the PSF and regularization operator is implemented using $p s f 2 o t f$, while for images, it is implemented using $f f t 2$ function.

Algorithm 1: Summary of the multichannel recursive filtering for image restoration.

Let $\mathbf{f}^{(n)} n=1,2, \ldots, K$ be the estimated image from the observed data $\left\{\mathbf{g}_{1}, \ldots, \mathbf{g}_{n}\right\}$ at the $n$th recursive step. The estimated $\mathbf{f}^{(n+1)}$ can be updated by using the information contained in the newly received observation $\mathbf{g}_{n+1}$ through

$$
\mathbf{f}^{(n+1)}=\mathbf{f}^{(n)}+\mathbf{u}^{(n+1)}
$$

where $\mathbf{u}^{(n+1)}$ is the update term, to be derived in the later part of this section. In the development of MRF, we incorporate a forgetting factor $\lambda$ into the cost function of (4) to ensure that the data in the distant past are assigned less emphasis [16]. Thus, we can reexpress (4) in the matrix-vector notation with the forgetting factor $\lambda$ as

$$
\mathbf{f}^{(n)}=\min _{\mathbf{f}} \sum_{i=1}^{n} \lambda^{n-i}\left(\left\|\mathbf{g}_{i}-\mathbf{H}_{i} \mathbf{f}\right\|^{2}+\alpha_{i}\|\mathbf{C} \mathbf{f}\|^{2}\right)
$$

where $\mathbf{H}_{i}$ and $\mathbf{C}$ are the block-circulant matrices that are constructed from $\mathbf{h}_{i}$ and $\mathbf{c}$, respectively. The selection issue of $\lambda$, c, $\alpha_{i}$ will be discussed in the next section. It is worth mentioning that the special case of $\lambda=1$ means infinite memory as the effect of past data is not attenuated. In contrast, the exponentially decaying memory channel $(\lambda<1)$ is used in time-varying environment. The closed-form solution to the least squares problem in (10) using the pseudoinverse is given by

$$
\mathbf{f}^{(n)}=\left(\mathbf{R}^{(n)}\right)^{-1} \mathbf{r}^{(n)},
$$

where $\mathbf{R}^{(n)}=\sum_{i=1}^{n} \lambda^{n-i}\left(\mathbf{H}_{i}^{H} \mathbf{H}_{i}+\alpha_{i} \mathbf{C}_{i}^{H} \mathbf{C}_{i}\right)$ and $\mathbf{r}^{(n)}=$ $\sum_{i=1}^{n} \lambda^{n-i} \mathbf{H}_{i}^{H} \mathbf{g}_{i}$. The superscript $(\cdot)^{H}$ denotes the conjugate transpose.

When the cost function (10) includes the newly available $(n+1)$ th channel, the estimate $\mathbf{f}^{(n+1)}$ is given as

$$
\mathbf{f}^{(n+1)}=\left(\mathbf{R}^{(n+1)}\right)^{-1} \mathbf{r}^{(n+1)},
$$

where $\mathbf{R}^{(n+1)}=\lambda \mathbf{R}^{(n)}+\mathbf{H}_{n+1}^{H} \mathbf{H}_{n+1}+\alpha_{n+1} \mathbf{C}_{n+1}^{H} \mathbf{C}_{n+1}$ and $\mathbf{r}^{(n+1)}=$ $\lambda \mathbf{r}^{(n)}+\mathbf{H}_{n+1}^{H} \mathbf{g}_{n+1}$. The estimate $\mathbf{f}^{(n+1)}$ will depend on the $(n+$ 1)th identified blur $\mathbf{h}_{n+1}$, which is estimated from the blur identification step explained in the next subsection.

Substituting (9) and (11) into (12), the update term $\mathbf{u}^{(n+1)}$ is given as

$$
\begin{aligned}
\mathbf{u}^{(n+1)}= & \left(\mathbf{R}^{(n+1)}\right)^{-1} \\
& \times\left(\mathbf{H}_{n+1}^{H} \mathbf{g}_{n+1}-\left(\mathbf{H}_{n+1}^{H} \mathbf{H}_{n+1}+\alpha_{n+1} \mathbf{C}_{n+1}{ }^{H} \mathbf{C}_{n+1}\right) \mathbf{f}^{(n)}\right) .
\end{aligned}
$$

Hence, the $(n+1)$ th least squares estimate $\mathbf{f}^{(n+1)}$ can be computed recursively from its previous estimate $\mathbf{f}^{(n)}$ using (9) and (17). However, the matrix $\left(\mathbf{R}^{(n+1)}\right)^{-1}$ cannot be computed readily from $\left(\mathbf{R}^{(n)}\right)^{-1}$ due to the huge computation cost associated with the inversion of the matrix $\left(\lambda \mathbf{R}^{(n)}+\mathbf{H}_{n+1}^{H} \mathbf{H}_{n+1}+\right.$ $\left.\alpha_{n+1} \mathbf{C}_{n+1}{ }^{H} \mathbf{C}_{n+1}\right)^{-1}$ (dimension of $M N \times M N$, where $M \times N$ is the size of the image). To address this issue, we exploit the diagonalization properties of the 2D-DFT for block-circulant matrix. The recursive filtering in spatial domain is transformed into the DFT domain. The proposed MRF image restoration is derived and summarized in Algorithm 1 where the superscript " $\sim$ " is used to denote the signal in the frequency domain, and $\boldsymbol{\omega}=\left(\omega_{x}, \omega_{y}\right)$ is used to denote the frequency pair along the $X$ - and $Y$-axes, respectively.

\subsection{Blur identification}

Blur identification is a challenging problem in blind image restoration, involving the estimation of its support size and coefficients. Blur support estimation is analogous to filter order estimation in one-dimensional (1D) signal processing, albeit the problem is in $2 \mathrm{D}$ spatial domain in this context $[17,18]$. In this work, we use the minimum cyclic shift 
(i) For the $i$ th channel $(i=1,2, \ldots, K)$, initialize the conjugate vector by setting

$$
\mathbf{q}^{(0)}=-\nabla_{\mathbf{h}_{i}} J^{(0)}
$$

(ii) At the $(n+1)$ th CGO iteration, $n=0,1, \ldots$,

update the $n$th iteration blur estimate:

$$
\mathbf{h}_{i}^{(n+1)}=\mathbf{h}_{i}^{(n)}+\eta^{(n)} \mathbf{q}^{(n)}, \quad \text { where } \eta^{(n)}=\frac{\left\|\nabla_{\mathbf{h}_{i}} J^{(n)}\right\|^{2}}{\left\|\mathbf{q}^{(n)} * \mathbf{f}\right\|^{2}+\beta_{i}\left\|\mathbf{d}_{i} * \mathbf{q}^{(n)}\right\|^{2}} .
$$

Update the $n$th conjugate vector:

$$
\mathbf{q}^{(n+1)}=-\nabla_{\mathbf{h}_{i}} J^{(n)}+\rho^{(n)} \mathbf{q}^{(n)}, \quad \text { where } \rho^{(k)}=\frac{\left\|\nabla_{\mathbf{h}_{i}} J^{(n+1)}\right\|^{2}}{\left\|\nabla_{\mathbf{h}_{i}} J^{(n)}\right\|^{2}} .
$$

Repeat until convergence or a maximum number of iterations is reached.

Algorithm 2: Summary of conjugate gradient optimization for blur identification.

correlation (MCSC) criterion in [17] to perform blur support estimation. The cost function involved in the blur coefficients estimation is given by

$$
\begin{aligned}
& J\left(\mathbf{h}_{1}, \ldots, \mathbf{h}_{K} \mid \mathbf{f}\right) \\
& \quad=\sum_{i=1}^{K}\left(\left\|\mathbf{g}_{i}-\mathbf{h}_{i} * \mathbf{f}\right\|^{2}+\beta_{i}\left\|\mathbf{d}_{i} * \mathbf{h}_{i}\right\|^{2}\right) .
\end{aligned}
$$

The gradient of $J(\mathbf{h} \mid \mathbf{f})$ with respect to $\mathbf{h}_{i}\{i=1,2, \ldots, K\}$ is given by

$$
\begin{aligned}
\nabla_{\mathbf{h}_{i}} J(x, y)= & 2\left[\mathbf{h}_{i}(x, y) * \mathbf{f}(x, y)-\mathbf{g}_{i}(x, y)\right] * \mathbf{f}(-x,-y) \\
& +2 \beta_{i}\left[\mathbf{d}_{i}(x, y) * \mathbf{h}_{i}(x, y)\right] * \mathbf{d}_{i}(-x,-y) .
\end{aligned}
$$

Conjugate gradient optimization (CGO) is used to minimize the cost function in (17). CGO utilizes conjugate direction instead of local gradient to search for the minima. Therefore, it can achieve faster convergence when compared with steepest descent method. It also requires less storage requirement and computational complexity when compared with Quasi-Newton method. Conjugate gradient optimization is ideal in this application as the given Hessian matrices are sparse, leading to fast convergence in small number of iterations. The mathematical formulations of blur identification based on conjugate gradient optimization are derived in Algorithm 2.

\subsection{Schematic overview}

The schematic overview of the proposed algorithm is given in Figure 1 . The idea is to alternately minimize the cost function with respect to the common $\mathbf{f}$ and the PSFs $\mathbf{h}_{i}$. The flowchart consists of two key steps. The first step performs recursive image restoration to yield $\mathbf{f}^{(i)}$ using $\mathbf{f}^{(i-1)}, \mathbf{R}^{(i-1)}$, and the new data of the $i$ th-channel. The second step performs blur identification using the conjugate gradient minimization to reach

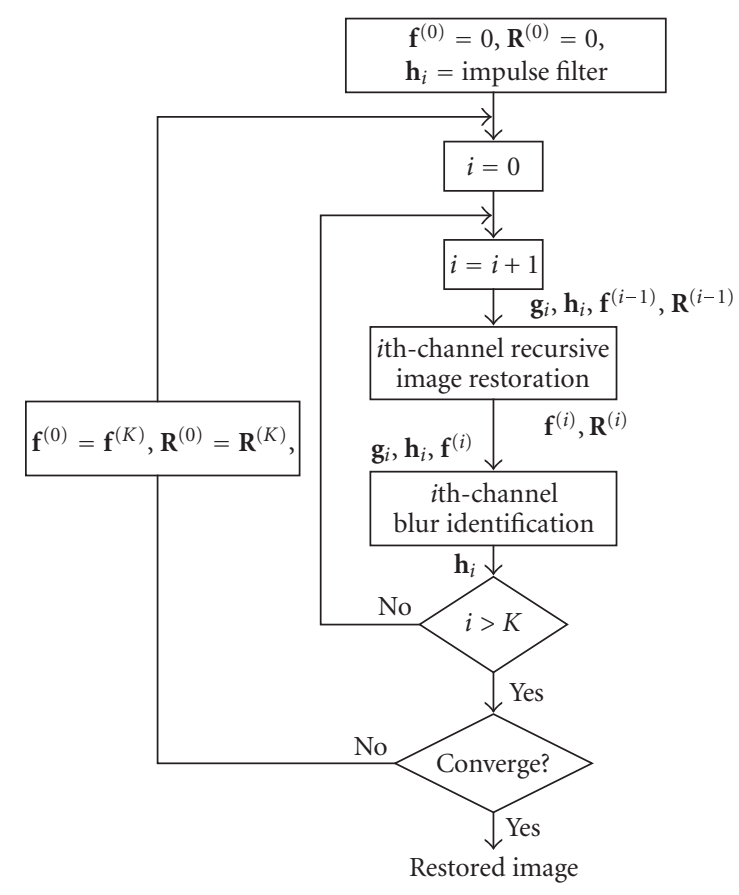

FIGURE 1: Schematic diagram of the proposed algorithm.

the optimal solution $\mathbf{h}_{i}$. Let $K$ be the total number of channels, the inner loop of the procedure will run these two steps alternately until data from all $K$ channels have been computed. Unlike recursive filtering in 1D adaptive filter design, multichannel image restoration does not have hundreds of measurements. Therefore, we propose to reuse the estimates $\mathbf{f}^{(0)}=\mathbf{f}^{(K)}, \mathbf{R}^{(0)}=\mathbf{R}^{(K)}$ from previous iteration in the outer loop to reiterate the inner loop till the convergence is reached. 
The contributions of the proposed technique, therefore, include the following. (i) As opposed to other multichannel restoration algorithms, it does not require all the data to be available simultaneously as recursive filtering updates the estimate based on first-come-first-served basis. (ii) All the operations of MRF for image-domain minimization are conducted in the frequency domain through DFT, hence efficiently reduce the computational cost. (iii) It incorporates a forgetting factor to discard the old unreliable estimates, hence achieving better convergence performance.

\section{ISSUES ON PARAMETER SELECTION}

\subsection{Regularization parameters and operators}

The regularization framework is instrumental in providing satisfactory results in image restoration. Let $\mathbf{e}^{(n)}$ denote the residual error between $\mathbf{f}^{(n)}$ in (11) and the original image $\mathbf{f}$ in (1). In the DFT domain, $\mathbf{e}^{(n)}$ is given by

$$
\begin{aligned}
\tilde{\mathbf{e}}^{(n)}(\boldsymbol{\omega})= & \widetilde{\mathbf{f}}^{(n)}(\boldsymbol{\omega})-\tilde{\mathbf{f}}(\boldsymbol{\omega}) \\
= & \frac{\sum_{i=1}^{n} \lambda^{n-i} \mathbf{H}_{i}^{H}(\boldsymbol{\omega}) \widetilde{\mathbf{n}}_{i}(\boldsymbol{\omega})}{\sum_{i=1}^{n} \lambda^{n-i}\left(\left|\mathbf{H}_{i}(\boldsymbol{\omega})\right|^{2}+\alpha_{i}|\widetilde{\mathbf{C}}(\boldsymbol{\omega})|^{2}\right)} \\
& -\frac{\mathbf{f}(\boldsymbol{\omega}) \sum_{i=1}^{n} \lambda^{n-i} \alpha_{i}|\widetilde{\mathbf{C}}(\boldsymbol{\omega})|^{2}}{\sum_{i=1}^{n} \lambda^{n-i}\left(\left|\mathbf{H}_{i}(\boldsymbol{\omega})\right|^{2}+\alpha_{i}|\widetilde{\mathbf{C}}(\boldsymbol{\omega})|^{2}\right)} .
\end{aligned}
$$

It can be observed that the error consists of two parts: the noise and the image terms. The first part is the noise term, which will be large for small $\mathbf{H}_{i}(\boldsymbol{\omega})$ if there is no regularization term $\alpha_{i}|\widetilde{\mathbf{C}}(\boldsymbol{\omega})|^{2}$. Thus, $\alpha_{i}\left|\widetilde{\mathbf{C}}_{i}(\boldsymbol{\omega})\right|^{2}$ will reduce the impact of noise term. However, this is at the cost of producing a small bias to the actual image. In order to make $\mathbf{e}^{(n)}$ as small as possible, a reasonable compromise needs to be reached between these two terms by careful determination of regularization parameter and operator. Previous work on the selection of the regularization parameter includes set theoretic approach and generalized cross-validation [19]. We follow the idea of set theoretic in [19] to estimate the regularization parameters $\alpha_{i}$ and $\beta_{i}$ :

$$
\alpha_{i}=\frac{\varepsilon_{i}^{2}}{\gamma^{2}} \approx \frac{M N \sigma_{i}^{2}}{\|\mathbf{c} * \mathbf{f}\|^{2}}, \quad \beta_{i}=\frac{\varepsilon_{i}^{2}}{\delta_{i}^{2}} \approx \frac{M N \sigma_{i}^{2}}{\left\|\mathbf{d}_{i} * \mathbf{h}_{i}\right\|^{2}},
$$

where $\varepsilon_{i}, \gamma, \delta_{i}$ are the upper bounds related to the noise, image, and PSF terms in (2). $\sigma_{i}^{2}$ is the noise variance in the $i$ thchannel, which can be estimated from the smooth regions of the image. Equation (20) suggests a rule of thumb to choose reasonable regularization parameters based on the noise, image, and PSF conditions. In the experiments, the regularization parameters $\alpha_{i}$ and $\beta_{i}$ are initialized and remained constant during the AM procedure. It is also possible to use the estimated $\hat{\sigma}_{i}, \hat{\mathbf{f}}$, and $\hat{\mathbf{h}}$ to provide an order-of-magnitude estimate for the regularization parameters $[12,14,19]$. The simulation results show that the algorithm is robust towards different regularization parameters so long as they fall within a reasonable range.
As PSF is generally a low-pass filter, c should be taken as a high-pass filter (or simply as an identity matrix $\mathbf{C}=\mathbf{I}$ ), which imposes smooth constraints on the images. The analysis on the regularization operator $\mathbf{d}_{i}$ is similar to $\mathbf{c}$. In the appendix, an analysis on how the regularization result of (10) is affected by the error in the PSFs is outlined.

\subsection{Forgetting factor}

The introduction of forgetting factor is centered on the observation that when the estimated image converges to the original one, the identified blur will approach the actual PSF in the alternating minimization scheme. It can be observed from (10) that if the forgetting factor is $0 \leq \lambda<1$, the scheme will diminish older, less reliable estimated $\mathbf{h}_{i}$, and favor later, more updated estimate. Generally speaking, the forgetting factor plays the role as an adaptive weight to ensure that the data in the distant past are assigned less emphasis. Even though the optimal value of the forgetting factor can be derived theoretically using constrained optimization technique such as Lagrange multiplier method, this optimal value is a function of the original image, PSF, and noise, of which we have no prior knowledge. Therefore, it is tedious and impractical to estimate this optimal value during iterative minimization. It should also be noted from the experiments that the proposed method will produce reasonable results so long as the forgetting factor is within a reasonable range. Therefore, estimation of exact optimal value is not required. In this work, we let $\lambda=\zeta^{1 / K}$, where $\zeta$ is the memory attenuation rate, $K$ is the number of channels. For example, $\lambda=0.3^{1 / K}$ means that the current channel has a weight of only $30 \%$ left in its next iteration.

\section{EXPERIMENTAL RESULTS}

\subsection{Multichannel blind restoration under noisy conditions}

The effectiveness of the proposed method is illustrated under different blurring conditions. For performance evaluation, peak signal-to-noise ratio (PSNR) is chosen as the objective performance metric. In Figure 2(a), the original "Board" image of size $256 \times 256$ is selected as the test image. The image is blurred by four $5 \times 5$ Gaussian blurs:

$$
h(i, j)=a \exp \left(-\frac{\left(i^{2}+j^{2}\right)}{2 \sigma^{2}}\right), \quad i, j=0, \pm 1, \pm 2,
$$

corresponding to different values of $\sigma_{i}=\{2.0,2.5,3.0,3.5\}$, where $\sigma$ is the standard deviation of the Gaussian blur. The parameter $a$ is the normalization constant which ensures the PSF coefficients sum up to 1 . Further, the blurred image is degraded under different noise levels to produce different SNR values $\{30 \mathrm{~dB}, 33 \mathrm{~dB}, 36 \mathrm{~dB}, 40 \mathrm{~dB}\}$. Through this, we can simulate four acquisition channels with variable blurring functions and noise levels, as shown in Figure 2(b).

The proposed MRF algorithm is run to perform blind image restoration. All the degraded images are firstly 


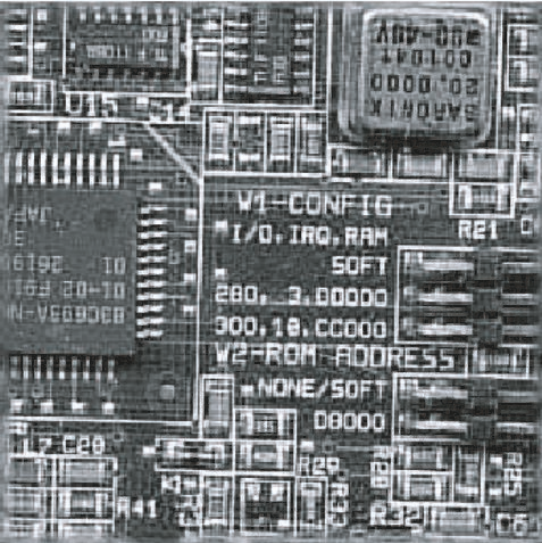

(a)

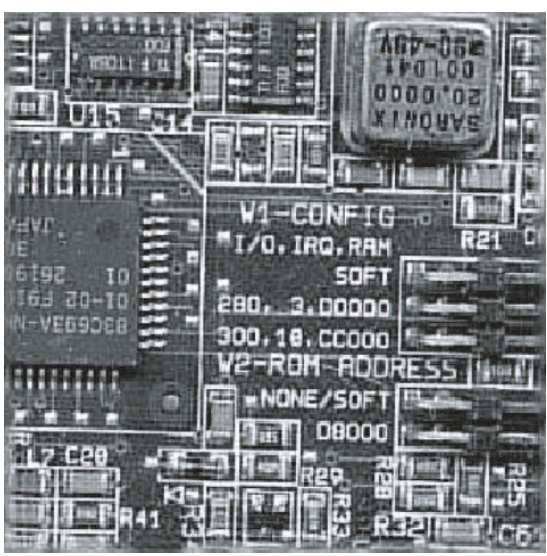

(c)

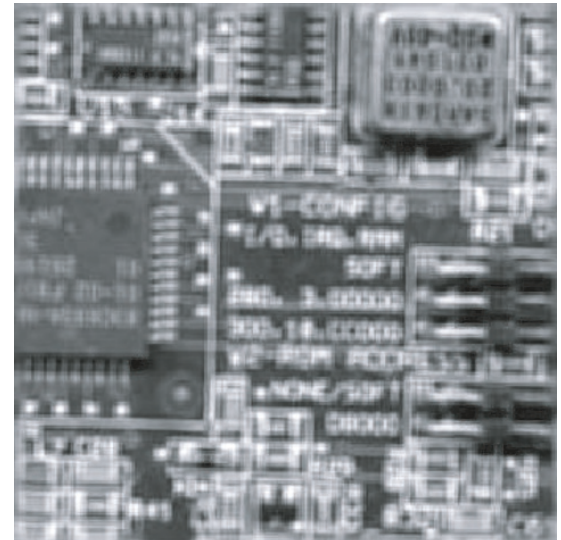

(b)

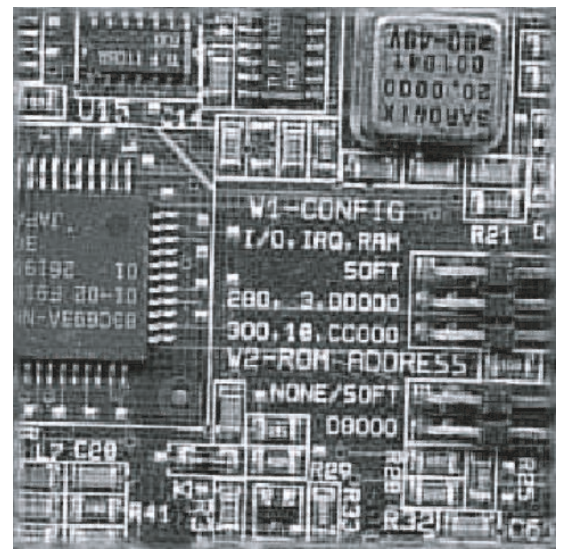

(d)

Figure 2: Multichannel blind image restoration results. (a) Original "Board" image. (b) A sampled blurred image out of the four degraded images. (c) Restored image using the proposed MRF algorithm with identity regularization operator. (d) Restored image using the proposed MRF algorithm with Laplacian regularization operator.

preprocessed using the edgetaper function in Matlab to ensure that the images are circularly symmetric. The forgetting factor is taken as $\lambda=\zeta^{1 / K}$, where $\zeta=0.05$ and $K=4$. The regularization parameters are calculated according to (20), while the regularization operators are simply taken as identity matrix or high-pass filter. The outer loop iteration number is set to 10, while the CGO iteration for blur identification is 5 .

The restored image using the proposed algorithm is shown in Figures 2(c) and 2(d). Figure 2(c) is the restored image with identity regularization operator, while Figure 2(d) is the restored image with Laplacian filter. It is observed that the approach is effective in recovering detailed information, as demonstrated by the clear numbers on the board. The satisfactory subjective inspection of the image is supported by objective performance measure as our method offers a PSNR of $21.93 \mathrm{~dB}$ in Figure 2(c) and $22.43 \mathrm{~dB}$ in Figure 2(d), compared to $12.46 \mathrm{~dB}$ for the degraded images. Empirical results show that the proposed algorithm is not sensitive to the exact choice of regularization operators so long as they are reasonable. As the restored image with Laplacian operator offers better PSNR value, we will use Laplacian regularization operator for the next experiments.

\subsection{Comparison with other multichannel restoration methods}

To further evaluate the effectiveness of our algorithm, we compare the proposed algorithm with iterative multichannel restoration methods, namely CGO-AM [12], TV-AM [14], and Wiener filtering-alternating minimization (WFAM). The reason for choosing these methods for comparative study is that these methods decompose the multichannel blind deconvolution problem into two processes of image restoration and blur identification, which are iteratively optimized using alternating minimization. Their main difference lies in that the proposed method uses recursive filtering to update the results. CGO-AM method adopts conjugate gradient optimization (CGO) to minimize the image- and blur-domain cost functions, while total variation (TV) and 


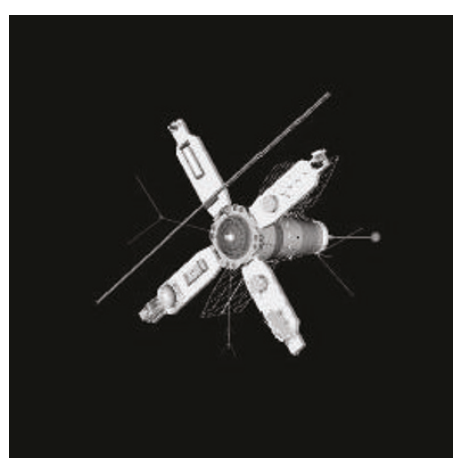

(a)

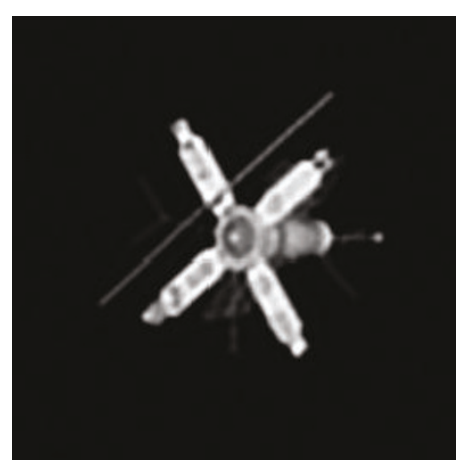

(d)

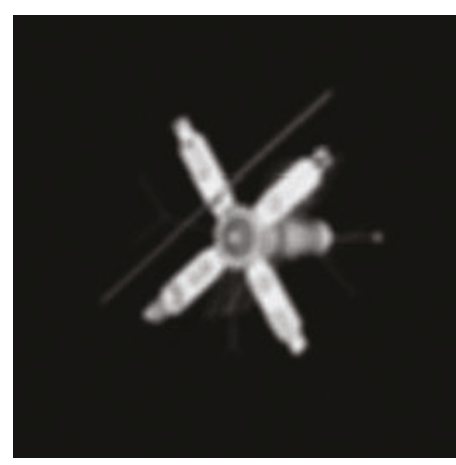

(b)

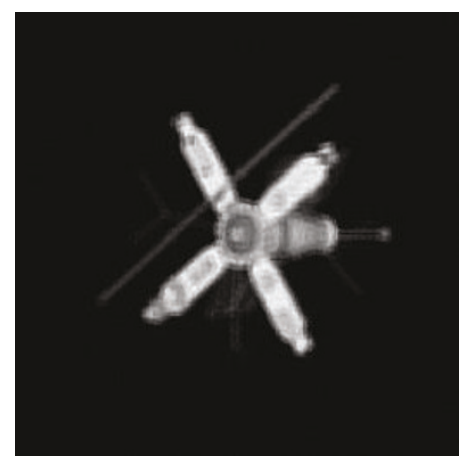

(e)

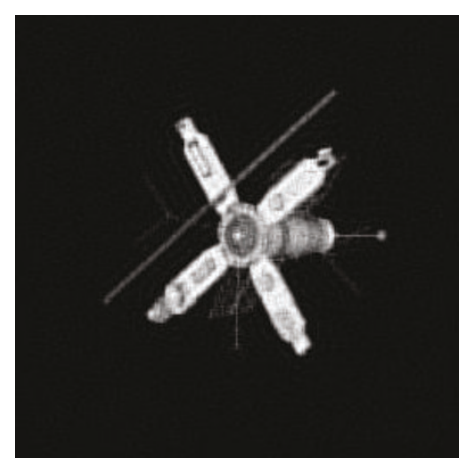

(c)

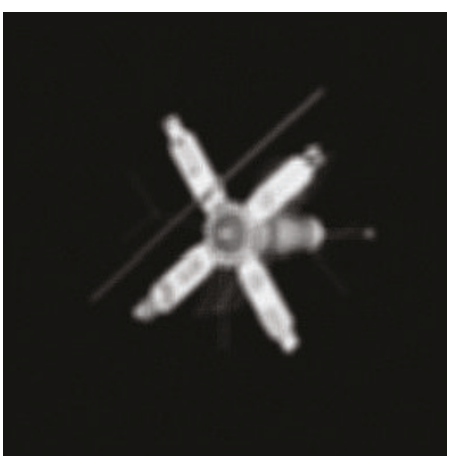

(f)

FIGURE 3: Comparison of different restoration results in $30 \mathrm{~dB}$ noise environment. (a) Original "satellite" image. (b) One of the three degraded images. (c) Restored image using the proposed MRF algorithm. (d) Restored image using the CGO-AM algorithm. (e) Restored image using the TV-AM algorithm. (f) Restored image using the WF-AM algorithm.

null space of blur are incorporated into the TV-AM scheme. The parameter settings of CGO-AM and TV-AM are calculated according to $[12,14]$. We have tried different parameter assignments to determine the suitable setting for all methods. The approach of WF-AM is explained as follows. The recursive filtering of image-domain cost function of the proposed method is similar to Wiener filtering. Since blind image restoration involves image restoration and blur identification, we replace the ith-channel recursive image restoration in Figure 1 by Wiener filtering. Conventional Wiener filtering [20] is performed using $\mathbf{f}(w)=\left(\mathbf{H}_{i}(\boldsymbol{\omega})^{T} \mathbf{H}_{i}(\boldsymbol{\omega})+\right.$ $\left.\alpha_{i}\right)^{-1} \mathbf{H}_{i}(\boldsymbol{\omega})^{T} \mathbf{g}_{i}(\boldsymbol{\omega})$, where $\alpha_{i}$ is the power spectrum ratio of the noise to the restored image. $\mathbf{H}_{i}$ and $\mathbf{g}_{i}$ are the PSF and degraded image in the $i$ th-channel, respectively. This outlines the WF-AM scheme to perform joint blur identification and image restoration. The iteration number is set to 10 for all methods.

The $256 \times 256$ "satellite image" shown in Figure $3(\mathrm{a})$ is degraded by different blurs under different noisy conditions ( $30 \mathrm{~dB}$ and $40 \mathrm{~dB}$ SNR noise). The proposed MRF, CGO-AM, TV-AM, and WF-AM are applied to the blurred image in Figure 3(b). The restored images are given in Figure 3(c)3 (f) for $30 \mathrm{~dB}$ noisy conditions, where PSNR is tabulated in Table 1 for $30 \mathrm{~dB}$ and $40 \mathrm{~dB}$ noisy conditions. On average, the proposed method yields $0.6 \mathrm{~dB}, 3.2 \mathrm{~dB}$, and $3.8 \mathrm{~dB}$ improvements over the CGO-AM, TV-AM, and WF-AM methods, respectively. By comparing the restored images shown in Figures 3(c)-3(f), it is clear that our approach is superior in preserving details of satellite. This is supported by objective performance measure as our method offers PSNR of $29.41 \mathrm{~dB}$, as opposed to $28.70 \mathrm{~dB}, 26.10 \mathrm{~dB}$, and $25.85 \mathrm{~dB}$ by the CGO-AM, TV-AM, and WF-AM methods, respectively. The proposed method utilizes recursive updating technique, coupled with forgetting term to prioritize newer, more recent estimates. In contrast, the error in previous estimate will be propagated in the CGO-AM method. On the other hand, the WF-AM method inherits no information from the older estimate as each channel restores the image independently. Further, the TV-AM method requires all the PSFs to be coprime. As this assumption is not satisfied in the experiment, the TV-AM method fails to provide satisfactory results.

The overall complexity is a combination of the convergence rate and the time complexity for each single iteration. The proposed method, generally, has faster convergence rate with moderate single-iteration complexity. To illustrate this, we try to compare the computational time of these methods using the same platform. The simulation environments of these methods are Windows XP, MATLAB 6.5, CPU P4$2.4 \mathrm{GHz}$, and $512 \mathrm{M}$ RAM. It takes 64 seconds in terms of the running time, as compared to 89 seconds, 179 seconds, and 55 seconds by the CGO-AM, TV-AM, and WF-AM methods, respectively. The reason that the proposed method is faster 
TABLE 1: Comparison of different restoration algorithms.

\begin{tabular}{l|ll|r}
\hline PSF & \multicolumn{2}{|l|}{ Gaussian $7 \times 7, \sigma_{i}=\{2.5,3.0,3.5\}$} & \multirow{2}{*}{ Time } \\
\hline Noise level & $30 \mathrm{~dB}$ & $40 \mathrm{~dB}$ & \\
\hline Proposed MRF & $28.58 \mathrm{~dB}$ & $29.41 \mathrm{~dB}$ & $64 \mathrm{~s}$ \\
\hline CGO-AM & $27.96 \mathrm{~dB}$ & $28.70 \mathrm{~dB}$ & $89 \mathrm{~s}$ \\
\hline TV-AM & $25.42 \mathrm{~dB}$ & $26.10 \mathrm{~dB}$ & $179 \mathrm{~s}$ \\
\hline WF-AM & $24.54 \mathrm{~dB}$ & $25.85 \mathrm{~dB}$ & $55 \mathrm{~s}$ \\
\hline
\end{tabular}

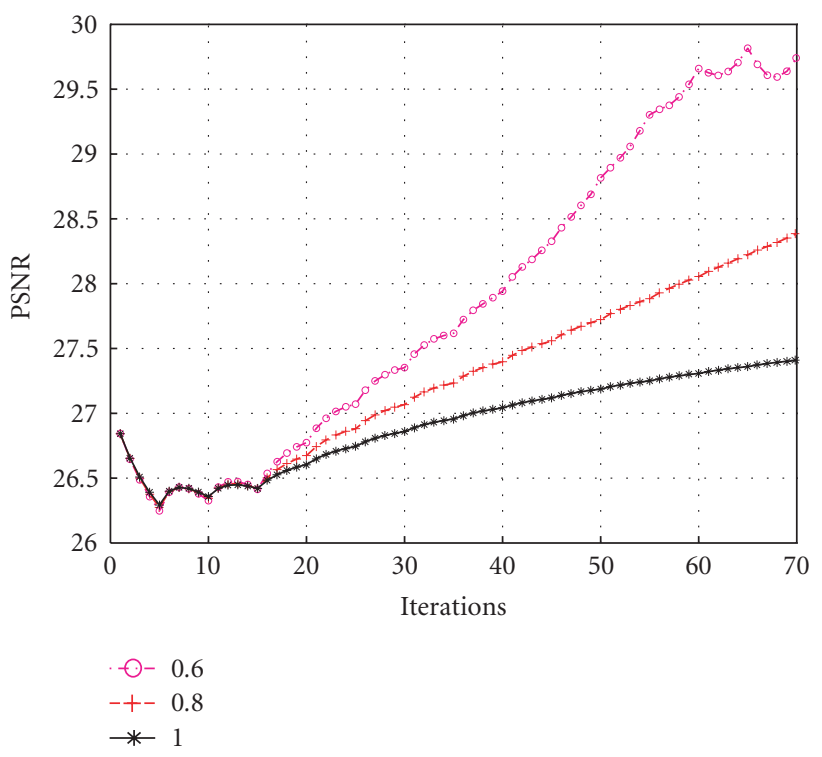

FIgURE 4: The profile of PSNR versus the number of iterations with different forgetting parameters $\lambda=\zeta^{1 / 5}$, where $\zeta=\{0.6,0.8,1.0\}$.

than the CGO-AM and TV-AM methods is due to efficient recursive updating of the images.

\subsection{Impact of forgetting factor}

To study the impact of forgetting factor experimentally, the proposed MRF algorithm is run with different forgetting factors. The $256 \times 256$ "Lena" image is degraded by $7 \times 7$ Gaussian blurs with $\sigma_{i}=\{2.0,2.3,2.6,2.9,3.2\}$. The regularization operators are standard Laplacian high-pass filter. Based on the 5 observed degraded images, the proposed MRF algorithm is repeated, but with different forgetting parameters $\lambda=\zeta^{1 / 5}$, where $\zeta=\{0.6,0.8,1.0\}$. The outer loop iteration number is set to 14 . As there are 5 channels, each inner loop iteration will update the image 5 times. The overall iteration of the recursive filtering is 70 . The profile of PSNR versus the number of iterations is plotted in Figure 4. It is observed that the curve tends to become flatter when the forgetting factor becomes larger. This indicates slower convergence rate. $\lambda=1$ means infinite memory as the effect of past data is not attenuated and this gives rise to slow convergence rate.

In this work, we simply use $\lambda=\zeta^{1 / K}$ to show that the algorithm can have different convergence rate for different forgetting factors. Good results can be obtained if we terminate the algorithm when the relative change in consecutive iteration is less than a predefined threshold. Further study on the effect of forgetting factor is interesting and we hope that this work will stimulate further investigation.

\section{CONCLUSION}

This paper proposes an iterative blind multichannel image restoration algorithm based on recursive filtering. The estimated image is recursively updated from its previous estimate using a regularization framework. It incorporates a forgetting factor to discard the old unreliable estimates, hence achieving better convergence performance. The proposed computational structure is novel and it makes the overall computation efficient, especially in the area of multichannel reconstruction. This allows the new method to be adopted readily in real-life applications. Experimental results show that it is effective in performing blind multichannel blind restoration.

\section{APPENDIX}

In this appendix, the error bound for the regularization scheme is studied. The error bound for the least squares solution to the overdetermined and underdetermined systems $\mathbf{A x}=\mathbf{b}$ is presented in [21]. To examine how the regularization solution is affected by the changes in $\mathbf{A}$ and $\mathbf{b}$, the regularized solution to the underdetermined/overdetermined systems $\mathbf{A x}=\mathbf{b}$ is investigated.

Proposition 1. Suppose $\mathbf{A} \in \mathbb{R}^{m \times n}, \delta \mathbf{A} \in \mathbb{R}^{m \times n}, 0 \neq \mathbf{b} \in$ $\mathbb{R}^{m}, \delta \mathbf{b} \in \mathbb{R}^{m}, 0<\alpha \in \mathbb{R}$, and $\operatorname{rank}(\mathbf{A})=m<n$. Let $\varepsilon=$ $\max \left\{\varepsilon_{A}, \varepsilon_{b}\right\}$, where $\varepsilon_{A}=\|\delta \mathbf{A}\|_{2} /\|\mathbf{A}\|_{2}$ and $\varepsilon_{b}=\|\delta \mathbf{b}\|_{2} /\|\mathbf{b}\|_{2}$. If $\mathbf{x}$ and $\hat{\mathbf{x}}$ are the regularization solutions that satisfy

$$
\begin{gathered}
\mathbf{x}=\left(\mathbf{A}^{T} \mathbf{A}+\alpha \mathbf{I}\right)^{-1} \mathbf{A}^{T} \mathbf{b}=\mathbf{A}^{T}\left(\mathbf{A A}^{T}+\alpha \mathbf{I}\right)^{-1} \mathbf{b}, \\
\hat{\mathbf{x}}=\left((\mathbf{A}+\delta \mathbf{A})^{T}(\mathbf{A}+\delta \mathbf{A})+\alpha \mathbf{I}\right)^{-1}(\mathbf{A}+\delta \mathbf{A})^{T}(\mathbf{b}+\delta \mathbf{b}),
\end{gathered}
$$

then

$$
\begin{aligned}
\frac{\|\hat{\mathbf{x}}-\mathbf{x}\|_{2}}{\|\mathbf{x}\|_{2}} \leq & \varepsilon\left\{\kappa_{2}(\mathbf{A})+\frac{\|\mathbf{A}\|_{2}}{2 \sqrt{\alpha}}\left(1+\frac{\|\mathbf{b}\|_{2}}{\|\mathbf{A}\|_{2}\left\|_{\mathbf{x}}\right\|_{2}}\right)\right\} \\
& +O\left(\varepsilon^{2}\right) .
\end{aligned}
$$

Proof. Let $\mathbf{E}$ and $\mathbf{q}$ be defined by $\delta \mathbf{A} / \varepsilon$ and $\delta \mathbf{b} / \varepsilon$. It follows that the solution $\mathbf{x}(t)$ to

$$
\left((\mathbf{A}+t \mathbf{E})^{T}(\mathbf{A}+t \mathbf{E})+\alpha \mathbf{I}\right) \mathbf{x}(t)=(\mathbf{A}+t \mathbf{E})^{T}(\mathbf{b}+t \mathbf{q})
$$

is continuously differentiable for all $t \in[0, \infty)$.

Define $\mathbf{P}_{1}=\mathbf{A}^{T} \mathbf{A}+\alpha \mathbf{I}$ and $\mathbf{P}_{2}=\mathbf{A} \mathbf{A}^{T}+\alpha \mathbf{I}$, we obtain

$$
\begin{gathered}
\mathbf{P}_{1}^{-1} \mathbf{A}^{T}=\mathbf{A}^{T} \mathbf{P}_{2}^{-1}, \\
\|\mathbf{x}\|_{2}=\left\|\mathbf{A}^{T} \mathbf{P}_{2}^{-1} \mathbf{b}\right\|_{2} \geq \sigma_{m}\left\|\mathbf{P}_{2}^{-1} \mathbf{b}\right\|_{2}, \\
\left\|\mathbf{P}_{1}^{-1}\right\|_{2}=\frac{1}{\alpha}, \\
\left\|\mathbf{P}_{2}^{-1}\right\|_{2}=\frac{1}{\left(\sigma_{m}^{2}+\alpha\right)}, \\
\left\|\mathbf{P}_{1}^{-1} \mathbf{A}^{T}\right\|_{2}=\max _{i} \frac{\sigma_{i}}{\left(\sigma_{i}^{2}+\alpha\right)} \leq 1 /(2 \sqrt{\alpha}),
\end{gathered}
$$

where $\sigma_{i}$ is the singular value of $\mathbf{A}$ and $\sigma_{1} \geq \sigma_{2} \geq \cdots \geq \sigma_{m}>0$. 
By differentiating (A.3) with respect to $t$ and setting $\mathrm{t}=0$ in the result, we obtain

$$
\dot{\mathbf{x}}(0)=\mathbf{P}_{1}^{-1} \mathbf{A}^{T}(\mathbf{q}-\mathbf{E x})+\alpha \mathbf{P}_{1}^{-1} \mathbf{E}^{T} \mathbf{P}_{2}^{-1} \mathbf{b} .
$$

Since $\mathbf{x}=\mathbf{x}(0), \hat{\mathbf{x}}=\mathbf{x}(\varepsilon)$, the error upper bound is given by

$$
\begin{aligned}
\frac{\|\hat{\mathbf{x}}-\mathbf{x}\|_{2}}{\|\mathbf{x}\|_{2}}= & \varepsilon \frac{\|\dot{\mathbf{x}}(0)\|_{2}}{\|\mathbf{x}\|_{2}}+O\left(\varepsilon^{2}\right) \\
\leq & \varepsilon\left\{\|\mathbf{A}\|_{2}\left\|\mathbf{P}_{1}^{-1} \mathbf{A}^{T}\right\|_{2}\left(\frac{\|\mathbf{q}\|_{2}}{\|\mathbf{A}\|_{2} \|_{\mathbf{x} \|_{2}}}+\frac{\|\mathbf{E}\|_{2}}{\|\mathbf{A}\|_{2}}\right)\right. \\
& \left.\quad+\frac{\alpha}{\sigma_{m}}\|\mathbf{A}\|_{2}\left\|\mathbf{P}_{1}^{-1}\right\|_{2} \frac{\left\|\mathbf{E}^{T}\right\|_{2}}{\|\mathbf{A}\|_{2}}\right\}+O\left(\varepsilon^{2}\right) \\
\leq & \varepsilon_{A} \kappa_{2}(\mathbf{A})+\frac{\|\mathbf{A}\|_{2}}{2 \sqrt{\alpha}}\left\{\varepsilon_{A}+\varepsilon_{b} \frac{\|\mathbf{b}\|_{2}}{\|\mathbf{A}\|_{2}\|\mathbf{x}\|_{2}}\right\} \\
& +O\left(\varepsilon^{2}\right)
\end{aligned}
$$

thereby establishing (A.2).

The extension of error bound to SIMO system in (10) when $\mathbf{C}=\mathbf{I}$ is straightforward by setting

$$
\begin{gathered}
\mathbf{A}=\left[\begin{array}{c}
\lambda^{n} \mathbf{H}_{1} \\
\lambda^{n-1} \mathbf{H}_{2} \\
\vdots \\
\mathbf{H}_{n}
\end{array}\right], \quad \delta \mathbf{A}=\left[\begin{array}{c}
\lambda^{n} \delta \mathbf{H}_{1} \\
\lambda^{n-1} \delta \mathbf{H}_{2} \\
\vdots \\
\delta \mathbf{H}_{n}
\end{array}\right], \\
\mathbf{b}=\left[\begin{array}{c}
\lambda^{n} \mathbf{g}_{1} \\
\lambda^{n-1} \mathbf{g}_{2} \\
\vdots \\
\mathbf{g}_{n}
\end{array}\right], \quad \delta \mathbf{b}=\left[\begin{array}{c}
\lambda^{n} \delta \mathbf{g}_{1} \\
\lambda^{n-1} \delta \mathbf{g}_{2} \\
\vdots \\
\delta \mathbf{g}_{n}
\end{array}\right], \\
\mathbf{x}=\mathbf{f}, \quad \alpha=\sum_{i=1}^{n} \lambda^{n-i} \alpha_{i} .
\end{gathered}
$$

In this case, the regularization operator is taken as impulse filter to simplify the analysis. Suppose that the estimated $\mathbf{H}_{i}$ converges to the actual PSF during the iterative MRF scheme, we will have $\delta \mathbf{H}_{n} \leq \cdots \leq \delta \mathbf{H}_{2} \leq \delta \mathbf{H}_{1}$. Therefore, $\delta \mathbf{A}$ in (A.7) will be reduced as the forgetting factor assigns less weight to larger error of $\delta \mathbf{H}_{i}$. In this sense, the upper bound error will be reduced progressively.

\section{REFERENCES}

[1] D. Kundur and D. Hatzinakos, "Blind image deconvolution," IEEE Signal Processing Magazine, vol. 13, no. 3, pp. 43-64, 1996.

[2] B. R. Hunt and O. Kuebler, "Karhunen-Loeve multispectral image restoration, part I: theory," IEEE Transactions on Acoustics, Speech, and Signal Processing, vol. 32, no. 3, pp. 592-600, 1984.

[3] S. U. Pillai and B. Liang, "Blind image restoration using a robust GCD approach," IEEE Transactions on Image Processing, vol. 8, no. 2, pp. 295-301, 1999.
[4] G. Harikumar and Y. Bresler, "Perfect blind restoration of images blurred by multiple filters: theory and efficient algorithms," IEEE Transactions on Image Processing, vol. 8, no. 2, pp. 202-219, 1999.

[5] G. B. Giannakis and R. W. Heath Jr., "Blind identification of multichannel FIR blurs and perfect image restoration," IEEE Transactions on Image Processing, vol. 9, no. 11, pp. 1877-1896, 2000.

[6] H.-T. Pai and A. C. Bovik, "On eigenstructure-based direct multichannel blind image restoration," IEEE Transactions on Image Processing, vol. 10, no. 10, pp. 1434-1446, 2001.

[7] N. P. Galatsanos, A. K. Katsaggelos, R. T. Chin, and A. D. Hillery, "Least squares restoration of multichannel images," IEEE Transactions on Signal Processing, vol. 39, no. 10, pp. 2222-2236, 1991.

[8] M. G. Kang and A. K. Katsaggelos, "Simultaneous multichannel image restoration and estimation of the regularization parameters," IEEE Transactions on Image Processing, vol. 6, no. 5, pp. 774-778, 1997.

[9] Y. Yang, N. P. Galatsanos, and H. Stark, "Projection-based blind deconvolution," Journal of the Optical Society of America A, vol. 11, no. 9, pp. 2401-2409, 1994.

[10] Y.-L. You and M. Kaveh, "Regularization approach to joint blur identification and image restoration," IEEE Transactions on Image Processing, vol. 5, no. 3, pp. 416-428, 1996.

[11] T. F. Chan and C. K. Wong, "Convergence of the alternating minimization algorithm for blind deconvolution," Linear Algebra and Its Applications, vol. 316, no. 1-3, pp. 259-285, 2000.

[12] T. W. S. Chow, X.-D. Li, and K.-T. Ng, "Double-regularization approach for blind restoration of multichannel imagery," IEEE Transactions on Circuits and Systems I: Fundamental Theory and Applications, vol. 48, no. 9, pp. 1075-1085, 2001.

[13] R. Molina, J. Mateos, A. K. Katsaggelos, and M. Vega, "Bayesian multichannel image restoration using compound Gauss-Markov random fields," IEEE Transactions on Image Processing, vol. 12, no. 12, pp. 1642-1654, 2003.

[14] F. Sroubek and J. Flusser, "Multichannel blind iterative image restoration," IEEE Transactions on Image Processing, vol. 12, no. 9, pp. 1094-1106, 2003.

[15] G. Panci, P. Campisi, S. Colonnese, and G. Scarano, "Multichannel blind image deconvolution using the Bussgang algorithm: spatial and multiresolution approaches," IEEE Transactions on Image Processing, vol. 12, no. 11, pp. 1324-1337, 2003.

[16] S. Haykin, Adaptive Filter Theory, Prentice-Hall, Upper Saddle River, NJ, USA, 4th edition, 2002.

[17] L. Chen and K.-H. Yap, "A soft double regularization approach to parametric blind image deconvolution," IEEE Transactions on Image Processing, vol. 14, no. 5, pp. 624-633, 2005.

[18] L. Chen and K.-H. Yap, "Efficient discrete spatial techniques for blur support identification in blind image deconvolution," IEEE Transactions on Signal Processing, vol. 54, no. 4, pp. 15571562, 2006.

[19] N. P. Galatsanos and A. K. Katsaggelos, "Methods for choosing the regularization parameter and estimating the noise variance in image restoration and their relation," IEEE Transactions on Image Processing, vol. 1, no. 3, pp. 322-336, 1992.

[20] H. C. Andrews and B. R. Hunt, Digital Image Restoration, Prentice-Hall, Upper Saddle River, NJ, USA, 1977.

[21] G. H. Golub and C. F. Van Loan, Matrix Computations, John Hopkins University Press, New York. NY, USA, 3rd edition, 1996. 
Li Chen received B.Eng. degree in industrial automation from Wuhan University of Technology, Wuhan, China, in 1999, the M.Eng. degree in control theory from Huazhong University of Science and Technology, Wuhan, China, in 2002, and the Ph.D. degree in information engineering from Nanyang technological University, Singapore, in 2006. He is currently a Research Associate at Nanyang Technological

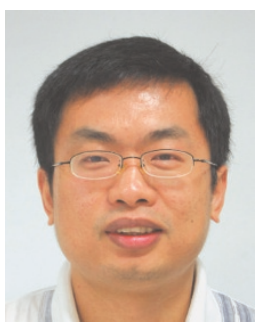
University, Singapore. His research interests include image processing, statistical pattern recognition, and computer vision.

Kim-Hui Yap received the B.Eng. and Ph.D. degrees in electrical engineering from the University of Sydney, Sydney, Australia, in 1998 and 2002, respectively. Currently, he is a Faculty Member at Nanyang Technological University, Singapore. His research interests include adaptive image processing, computational intelligence, and multimedia signal processing. He has more than thirty publications in various international jour-

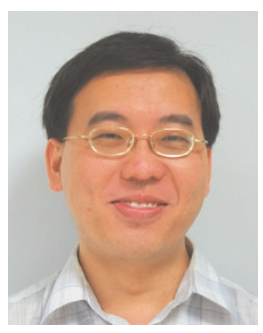
nals, conference proceedings, and book chapters. He is also the Editor of a book entitled Intelligent Multimedia Processing with Soft Computing by Springer-Verlag in 2005.

Yu He received B.Eng. degree in precision instrument and optoelectronics engineering from Tianjin University, Tianjin, China, in 2002. After that he worked as a Research and Develepment Engineer in the SAMSUNG Electronic Co. Ltd. (color TV) for two years. He is currently a Ph.D. student at Nanyang Technological University, Singapore. His research interests include image and video deconvolution, and super resolu-

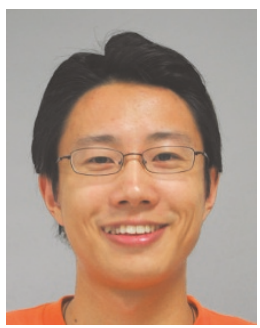
tion. 